\title{
Evaluación del rendimiento de semilla prebásica de tres variedades de papa (Solanum tuberosum) en un sistema aeropónico, distrito de Luya Viejo-región Amazonas
}

\section{Evaluation of the yield of prebasic seed of three potato varieties (Solanum tuberosum) in an aeroponic} system, district of Luya Viejo-Amazonas region

\author{
Luis Magallán-Maslucán ${ }^{1 *}$, Geysen Everson Angulo Cueva ${ }^{1}$, Lily del Pilar Juárez Contreras ${ }^{1}$, Gelver Silva Valqui ${ }^{1}$, Santos \\ Leiva-Espinoza ${ }^{1}$
}

\section{RESUMEN}

La aeroponía es la mejor alternativa para asegurar la disponibilidad de semilla de alta calidad. El presente trabajo de investigación, se llevó acabo en el distrito de Luya Viejo, provincia de Luya, Región Amazonas, durante los meses de junio a diciembre de 2017, con el objetivo de evaluar el rendimiento de semilla prebásica a nivel de invernadero, en tres variedades de papa (Solanum tuberosum) en un sistema aeropónico. Para ello se utilizó el diseño completo al azar (DCA), con tres tratamientos y cinco repeticiones. Para la comparación de medias de los resultados se utilizó la prueba de Tukey al $95 \%$ de confianza y para elprocesamiento de datos se empleó el software R. Asimismo los tratamientos evaluados fueron: T1 (variedad Yungay), T2 (variedad Huayro Amazonense) y T3 (variedad Canchán). Los tubérculos se desarrollaron en una cámara aeropónica conectada por aspersores para la distribución de la solución nutritiva y alimento de las mismas canalizado a través de sus raíces suspendidas en este medio, sin la necesidad de emplear un sustrato como es el caso de un sistema hidropónico. Así mismo, se evaluaron tres variables tales como la altura de planta, número de tubérculos por planta en cosecha y el peso de tubérculo por planta, de lo cual se alcanzó la mayor altura promedio de planta en el tratamiento T1 y la menor altura promedio de planta en el tratamiento T3. Sin embargo, en las variables del número de tubérculos planta en cosecha y peso del tubérculo por planta, no se reportó una diferencia significativa entre tratamientos, por lo que se determinó que, las tres variedades de papa (Solanum tuberosum) tomadas como objeto de estudio, expresan aptitud para ser utilizadas como fuente de propagación de semilla prebásica a nivel de Invernadero.

Palabras claves: Semilla prebásica, sistema aeropónico, variedad.

\begin{abstract}
Aeroponics is the best alternative to ensure the availability of high quality seed.The present research work was carried out in the district of Luya Viejo, province of Luya, Amazonas region, during the months of June to December 2017, with the objective of evaluating the yield of prebasic seed at greenhouse level, in three potato varieties (Solanum tuberosum) in the aeroponic system. For this, the complete randomized design (DCA) was used, with three treatments and five repetitions, for the comparison of the means of the results, the Tukey test was used at $95 \%$ confidence and the $\mathrm{R}$ software was used for data processing. Also the evaluated treatments were: T1 (variety Yungay), T2 (variety Huayro Amazonense) and T3 (variety Canchan). Tubers were developed in an aeroponic chamber connected by sprinklers for distribution of the nutrient solution and feed the same channeled through their roots suspended in this medium, without the need for a substrate such as a hydroponics system. Likewise, three variables were evaluated such as plant height, number of tubers per plant in harvest and tuber weight per plant, of which the highest average plant height was reached in treatment T1 and the lowest average height of plant in the T3 treatment. However, in the variables of the number of tubers plant in harvest and tuber weight per plant, no significant difference between treatments was reported, so it was determined that the three varieties of potato (Solanum tuberosum) taken as object of study, express aptitude to be used as a source of propagation of prebasic seed at the Greenhouse level.
\end{abstract}

Keywords: Prebasic seed, aeroponic system, variety. 


\section{INTRODUCCIÓN}

Las papas se propagan vegetativamente por tubérculos, lo que implica la disponibilidad de materiales de siembra libres de enfermedades. El acceso limitado a semillas de alta calidad es un problema endémico en países subdesarrollados como resultado de múltiples incidencias que aparecen durante la producción de semillas. La producción de semilla prebásica está respaldada por el cultivo de tejidos y generalmente requiere la utilización de técnicas de cultivo in vitro (Ahloowalia, 1999) para multiplicar plantas clónicas libres de enfermedades, rápidamente y cultivarlas bajo condiciones sanitarias (Ranalli, 1997). Sin embargo, es un procedimiento laborioso y costoso debido a la productividad reducida de los sistemas convencionales de multiplicación de semillas (Medeiros et al., 2002).

La producción de minitubérculos, con la utilización de sustratos de suelo o mezclas de varios compuestos sólidos está muy extendida en toda América Latina. Con frecuencia, los suelos se extraen de sitios vulnerables como el páramo de gran altitud o los ecosistemas de puna en Ecuador y Perú. Además, los sustratos se desinfectan utilizando calor o productos altamente tóxicos con un impacto negativo significativo sobre el medio ambiente y la salud humana (Gullino et al., 2003).

Se han establecido sistemas aeropónicos para la producción de semilla prebásica de la papa luego de una mayor demanda de métodos de producción de semillas de alta calidad más eficientes (Kang et al. 1996). Por lo tanto, la aeroponía o la aerohidroponía han desplazado a los sistemas tradicionales para la producción de minitubérculos (Chang et al., 2012).

En el sistema aeropónico, la producción de papa de siembra de categoría prebásica, debe partir necesariamente de un material de alta calidad libre de enfermedades y ser producido en invernadero, que es un sistema donde las raíces están expuestas en el aire, de manera continua o discontinua a un ambiente saturado de finas gotas de una solución nutritiva, y no requiere sustrato alguno ya que las raíces de las plantas se encuen- tran suspendidas en el aire y crecen dentro de contenedores vacíos y oscuros (Ritter y Relloso, 2000).

La semilla, es uno de los factores de mayor importancia para la producción agrícola. Una semilla de buena calidad aumenta la producción, productividad y optimiza el uso de insumos debido a una mayor uniformidad de emergencia y vigor de plantas (Velásquez, 2006).

En el Perú, la papa (Solanum tuberosum), es uno de los principales cultivos del país, dada su importancia económica y social, generando cada año aproximadamente 110000 puestos de trabajo permanentes (30 millones de jornales), y representando el $4 \%$ del Producto Bruto Interno (PBI) agrícola. Esto permite brindar al mundo casi 3000 variedades de papa, siendo la base de la alimentación de la población alto andina (Iparraguirre, 2017).

Hidalgo et al., 2009 informaron que el uso de semilla de tubérculo certificada en relación con todas las semillas sembradas en Perú fue $0.34 \%$ en 2005, 0.24\% en 2006 y $0.46 \%$ en 2007.

En este contexto, la aeroponía es una tecnología que contribuye a la propagación de semilla para los productos de Amazonas, por lo cual, en este estudio, se trazó el objetivo de determinar el mejor rendimiento de semilla prebásica entre las tres variedades de papa tales como Yungay, Huayro Amazonense y Canchán, a fin de reducir las pérdidas económicas por semilla y mejorar la producción de este cultivo en la Región Amazonas.

\section{MATERIALES Y MÉTODOS}

El invernadero aeropónico del distrito de Luya Viejo se encuentra a 3000 m.s.n.m. Presentó una variación de temperatura de $11^{\circ} \mathrm{Ca} 17^{\circ} \mathrm{C}$ durante el día y de $4{ }^{\circ} \mathrm{C}$ a $8{ }^{\circ} \mathrm{C}$ por las noches, que por lo contrario en el interior del invernadero se presentó una variación de temperatura durante el día de $17{ }^{\circ} \mathrm{C}$ a $25^{\circ} \mathrm{C}$ y de $4{ }^{\circ} \mathrm{C}$ a $9{ }^{\circ} \mathrm{C}$ durante las noches. Con una humedad relativa entre el 60 y $70 \%$ (Figura 1 ).

El centro de investigación, tuvo un área de $17,48 \mathrm{~m}^{2}$, con un total de 285 plantas de papa (95 plantas por 

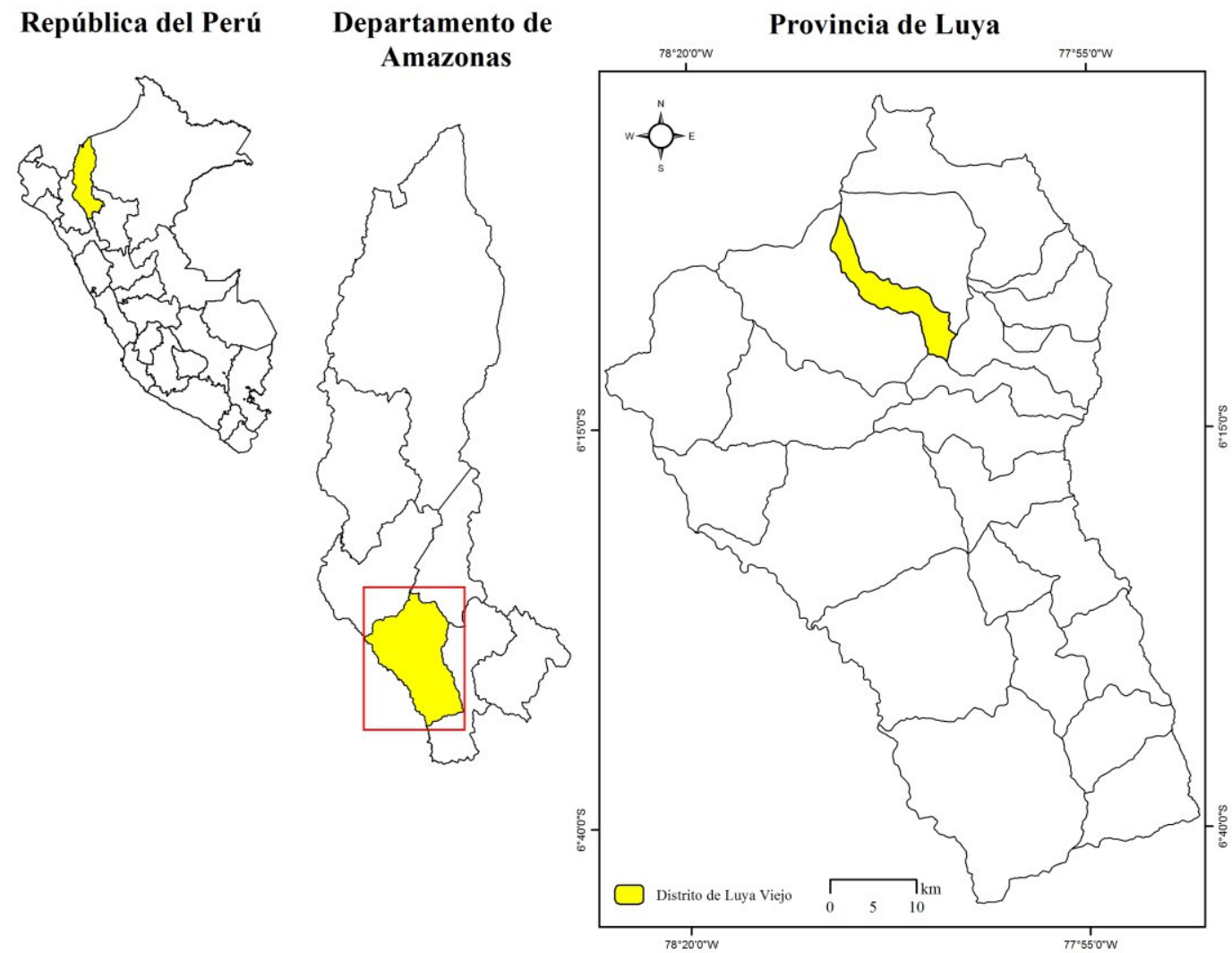

Tabla 1. Ubicación geográfica del área de estudio en el distrito de Luya Viejo (Provincia de Luya).

cada tratamiento), tres cajones aeropónicos (un cajón por cada tratamiento).

El material genético estudiado perteneció en su totalidad al cultivo de papa de las variedades Yungay, Huayro Amazonense y Canchán. Las plántulas se obtuvieron del INIA Baños del Inca, provincia y Región de Cajamarca, en magentas con agar como agente gelificante. Las plántulas se colocaron en el interior del invernadero para su aclimatación por dos días. Luego fueron trasplantadas a bandejas con arena, las cuales se desinfectaron previamente, permaneciendo por 20 días a fin de desarrollar sus raíces, para luego ser trasplantadas a los cajones aeropónicos, en los cuales se desarrollaron a lo largo de todo su periodo vegetativo y se realizaron todos los procesos de manejo tales como el aporque (un mes después del trasplante), las podas (cada cinco días después del trasplante), el colgado (después de dos semanas del trasplante y dos veces más cada 15 días), y finalmente la cosecha (en cinco momentos cada 14 días después de tres meses del trasplante)
Para el sistema aeropónico se construyeron tres cámaras oscuras (un cajón por cada tratamiento) para el desarrollo del radicular de las plantas (Figuras 2 y 3 ) y formación de los tubérculos, cada cajón aeropónico tuvo seis ventanas laterales y un sumidero para el retorno de la solución residual de nutrientes, un avanzado sistema de riego/drenaje permitiendo el control preciso de la solución de nutrientes, la formación de neblinas y ajustes. Esta solución nutritiva se bombeó continuamente desde un tanque. Las raíces de las plantas permanecieron en contacto directo con la neblina producida por los microaspersores (Iriman 02) alternativamente durante cinco minutos con interrupciones de 10 minutos, proporcionadas por la solución nutritiva que circulaba por la red de tubos conectados a los cajones aeropónicos y la solución residual de nutrientes retornó nuevamente al tanque. La calidad de la solución nutritiva se observó a diario durante todo el proceso teniendo como principales indicadores la $\mathrm{CE}$ con $13.52 \mathrm{Us} / \mathrm{cm}$ y el pH de 6.34 . 


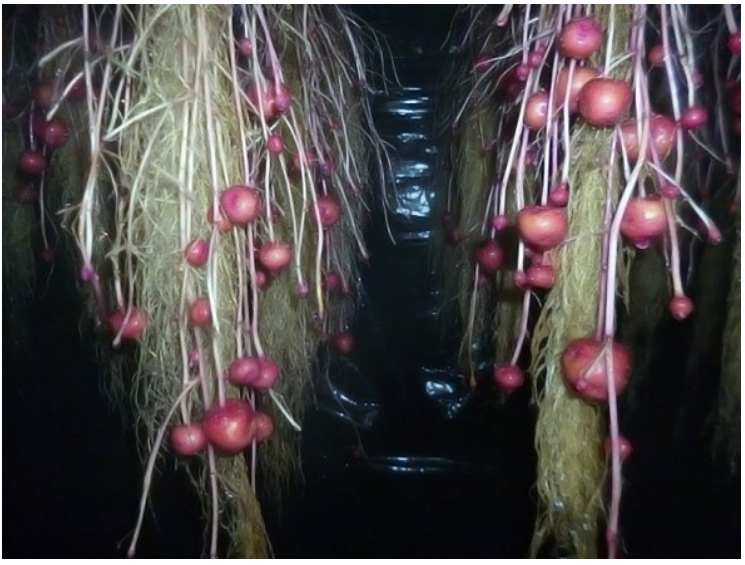

Figura 2. Producción de tuberculillos en el sistema aeropónico.

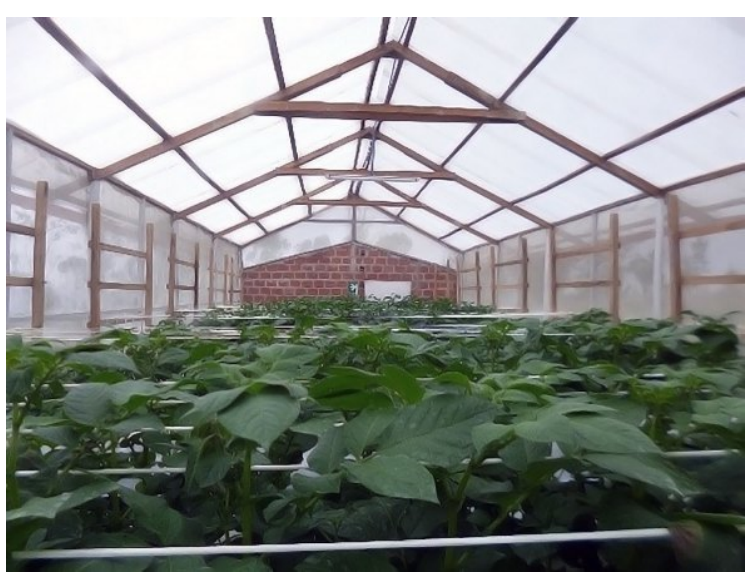

Figura 3. Diseño de los tratamientos en el sistema aeropónico

La solución nutritiva se preparó en un tanque de polietilieno de $1100 \mathrm{~L}$. El volumen de la solución en el tanque fue de $600 \mathrm{~L}$ el primer mes, y de $800 \mathrm{~L}$ a partir del segundo mes en adelante. Este nivel se mantuvo constataste a lo largo del periodo vegetativo. Además se realizó el cambio de la solución nutritiva cada 15 días en las madrugada a partir de las 6:00am para que las plantas no sufrieran un estrés hídrico por la falta de agua (Tabla 1).

Tabla 1. Componentes de la solución nutritiva

\begin{tabular}{c}
\hline Nitrato de amonio $\left(\mathrm{NH}_{4} \mathrm{NO}_{3}\right)$ \\
Nitrato de potasio $\left(\mathrm{KNO}_{3}\right)$ \\
Superfosfato triple de calci o $\left(\mathrm{Ca}\left(\mathrm{H}_{2} \mathrm{PO} 4\right)_{2}\right)$ \\
Sulfato de magnesio $\left.(\mathrm{Mg} \mathrm{SO})_{4}\right)$ \\
Fetrilón combi $(\mathrm{Fe}, \mathrm{Zn}, \mathrm{Cu}, \mathrm{Mn}, \mathrm{By} \mathrm{Mo})$ \\
\hline
\end{tabular}

En el primer mes (600 L de agua) se trabajó con $210 \mathrm{~g}$ de nitrato de amonio, $324 \mathrm{~g}$ de nitrato de potasio, $168 \mathrm{~g}$ de superfosfato triple de calcio, $144 \mathrm{~g}$ de sulfato de magnesio y $7,2 \mathrm{~g}$ fetrilón combi.
Así mismo, en el segundo mes (800 L de agua) se utilizaron $280 \mathrm{~g}$ de nitrato de amonio, $432 \mathrm{~g}$ de nitrato de potasio, $224 \mathrm{~g}$ de superfosfato triple de calcio, $192 \mathrm{~g}$ de sulfato de magnesio y 9,6 g fetrilón combi.

Y del tercer mes en adelante (800 L de agua) se trabajó con $140 \mathrm{~g}$ de nitrato de amonio, $432 \mathrm{~g}$ de nitrato de potasio, $224 \mathrm{~g}$ de superfosfato triple de calcio, $192 \mathrm{~g}$ de sulfato de magnesio y $9,6 \mathrm{~g}$ fetrilón combi.

La infraestructura del invernadero presentó algunas deficiencias, como la falta de malla rashel, para reducir la intensidad luminosa y evitar el marchitamiento de las hojas de la planta, por lo que se registró el ataque de rancha (Phytophtora infestans), enfermedad que se pudo controlar a penas se presentó en el cultivo, con la aplicación del fungicida Zampro DM a una dosis de 20 $\mathrm{mL}$ por mochila de $20 \mathrm{~L}$. en cinco momentos cada tres días y al final una aplicación foliar del fetrilón combi con una dosis de $20 \mathrm{~g}$ por mochila de $20 \mathrm{~L}$ después de tres días de la última aplicación del fungicida.

Se realizó un diseño completamente al azar (DCA), con tres tratamientos y cinco repeticiones. Cada tratamiento tuvo 95 plantas dispuestas en cinco hileras de 19 plantas (cada hilera era una repetición). Para el análisis de varianzas y la prueba de comparaciones múltiples utilizando la prueba Tukey al $95 \%$ de confianza, se empleó el software estadístico R.

De esta manera se realizaron cinco evaluaciones por cada variable. La altura de planta se midió con la ayuda de una regla de $30 \mathrm{~cm}$ y de una wincha para medidas superiores, desde el cuello de la planta hasta la yema terminal. Dichas evaluaciones se realizaron cada 20 días después del trasplante de las plántulas a los cajones aeropónicos.

En el número de tubérculos por planta por cosecha se registró el número de tubérculos obtenidos por planta en cada cosecha. Estas evaluaciones se realizaron cada 14 días después de tres meses del trasplante de las plántulas a los cajones aeropónicos.

Finalmente para el peso del tubérculo por planta, se registró el peso de cada tubérculo cosechado por planta en una balanza gramera cada 14 días después de la primera cosecha. 


\section{RESULTADOS}

En la variable altura de planta se realizaron cinco evaluaciones con un intervalo de 20 días después del trasplante de las plántulas a los cajones aeropónicos (Figura 4).
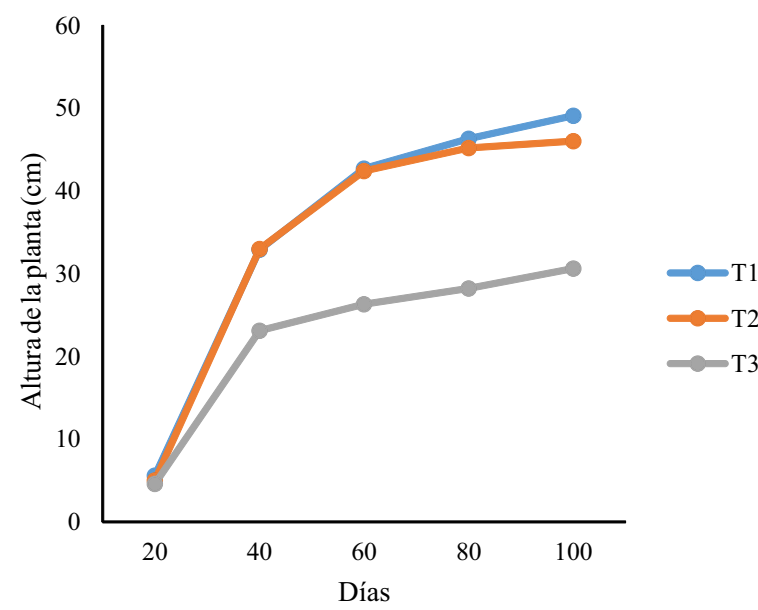

Figura 4. Desarrollo de la altura de planta durante el periodo vegetativo.

En la Figura 5, se detallan los valores promedios alcanzados en la variable altura de planta, en el cual se observa que el tratamiento T1 (variedad Yungay), con $53,47 \mathrm{~cm}$, fue el que alcanzó el mayor nivel de altura. Por lo contrario, en el tratamiento T3 (variedad Canchán), con $30,33 \mathrm{~cm}$, yalcanzó la menor altura promedio. Por otro lado el tratamiento T2 (variedad Huayro Amazonense), con 45,99 cm, alcanzó un valor medio respecto a los tratamientos anteriores.

Cuando se realizó la prueba de análisis de varianza, se encontró diferencia entre tratamientos. Tras lo cual se realizó la prueba de comparaciones múltiples Tukey al $95 \%$ de confianza, lo cual confirma que el tratamiento T1 es estadísticamente diferente al resto.

Así mismo, en la variable número de tubérculos por planta, se realizaron cinco evaluaciones con un intervalo de 14 días, y después de tres meses del trasplante de las plántulas a los cajones aeropónicos, por lo cual se obtuvieron los resultados expuestos en la Figura 6.

En la Figura 7 se detallan los valores promedios alcanzados en la variable número de tubérculos, en el cual se observa que los tratamientos T1 (variedad Yungay), con 2.83 unidades, el tratamiento T2

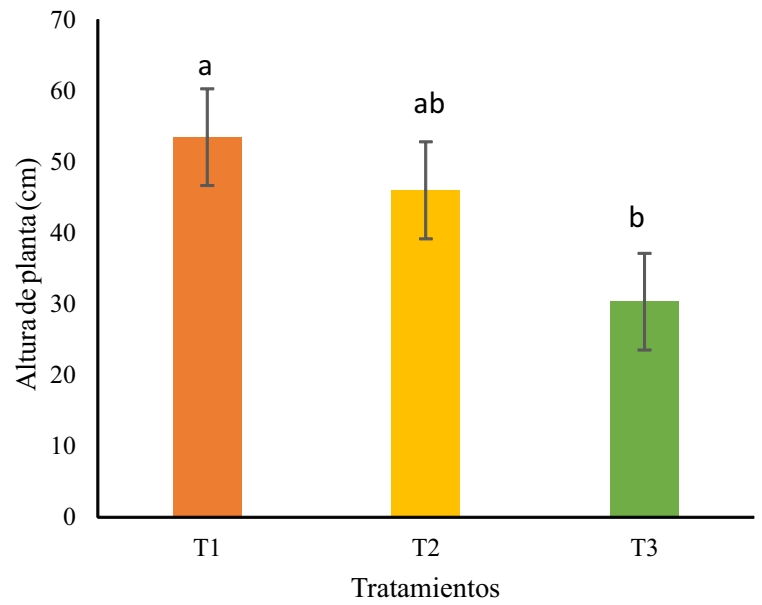

Figura 5. Distribución de la altura promedio por planta.

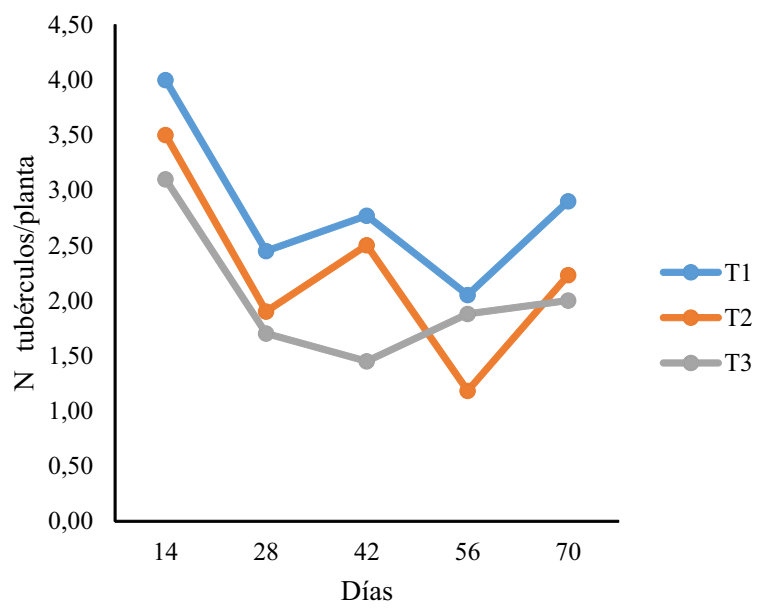

Figura 6. Desarrollo del número de tubérculos por planta.

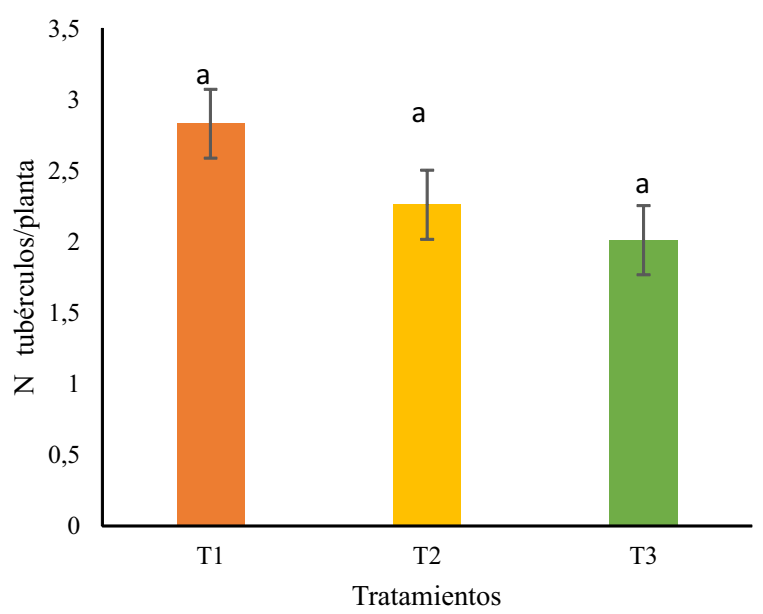

Figura 7. Distribución del número promedio de tubérculos por planta.

(Variedad Huayro Amazonense), con 2.26 unidades, y el tratamiento T3 (variedad Canchán) con 2.01 unidades producen similar número de tubérculos en cada cosecha.

Cuando se realizó la prueba de análisis de varianza no se encontraron diferencias entre tratamientos, tras lo 
cual se realizó la prueba de comparaciones múltiples de Tukey al $95 \%$ de confianza, a partir de la cual se confirma que los tratamientos T1, T2 y T3, son estadísticamente similares.

Finalmente, para la variable peso del tubérculo por planta, se realizaron cinco evaluaciones con un intervalo de 14 días, después de tres meses del trasplante de las plántulas a los cajones aeropónicos (Figura 8).

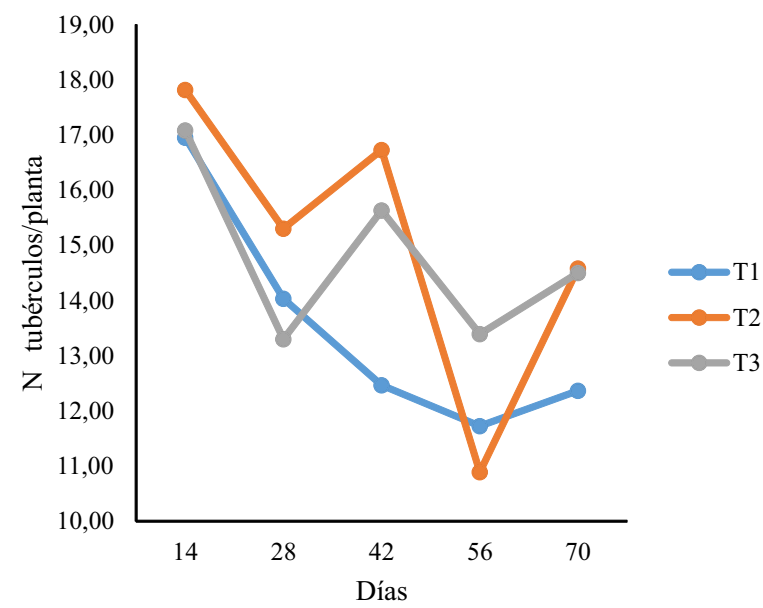

Figura 8. Desarrollo del peso del tubérculo.

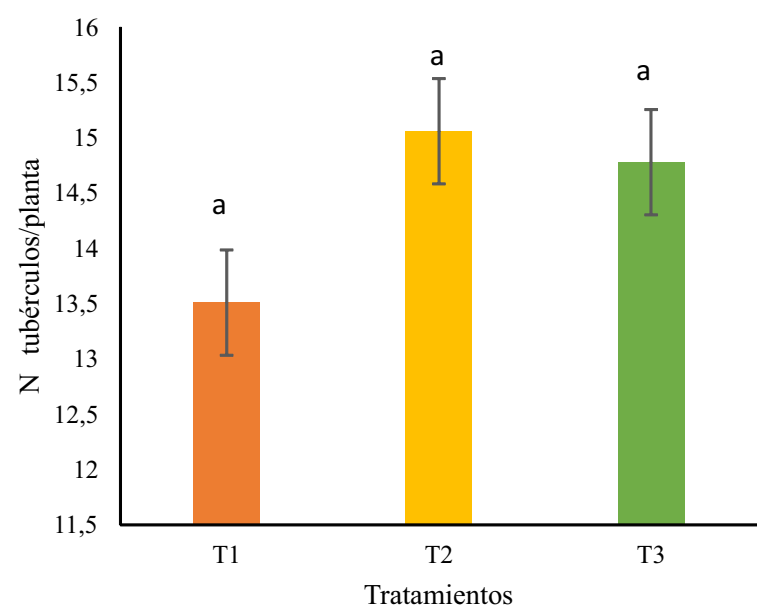

Figura 9. Distribución del peso promedio del tubérculo.

En la Figura 9, se detallan los valores promedios alcanzados en la variable peso del tubérculo, en el cual se observa que, los tratamientos T1 (variedad Yungay), con $13.51 \mathrm{~g}$, el tratamiento T2 (variedad Huayro Amazonense), con $15.06 \mathrm{~g}$, y el tratamiento T3 (variedad Canchán) con $14.78 \mathrm{~g}$ producen tubérculos con pesos similares.

Cuando se realizó la prueba de análisis de varianza, no se encontró diferencia entre tratamientos, tras lo cual se realizó la prueba de comparaciones múltiples Tukey al $95 \%$ de confianza, lo cual confirma que los tratamientos T1, T2 y T3, son estadísticamente similares.

\section{IV.DISCUSIÓN}

Los resultados alcanzados en la variable altura de planta, tienen una diferencia significativa principalmente entre los tratamientos $\mathrm{T} 1$ y T3, donde el promedio del tratamiento T1, fue mayor en un $17.83 \%$ del tratamiento $\mathrm{T} 3$, y relativamente superior al tratamiento $\mathrm{T} 2$ en un $5.76 \%$. Estos resultados son inferiores a lo presentado por Chang et al. (2011), quienes realizaron una investigación en el "Crecimiento y respuesta de rendimiento de tres cultivares de papa de crecimiento aeropónico (Solanum tuberosum L.) a diferente conductividad eléctrica de la solución de nutrientes", Así mismo, son inferiores a lo estudiado por Chango (2012) en su trabajo "Producción de semilla prebásica utilizando biol en un sistema aeropónico”. En tal contexto, se puede decir que la diferencia con ambos casos podría deberse a la presencia de Phytophtora infestans, el cual retardó el crecimiento de las plantas. Además, en los trabajos comparados, se probaron diferentes soluciones nutritivas y conductividad eléctrica, por lo cual las plantas expresaron diferentes comportamientos en su crecimiento.

Asimismo, en la variable número de tubérculos por planta en cosecha no se encontró diferencia significativa entre los tratamientos. Sin embargo el tratamiento $\mathrm{T} 1$, fue superior numéricamente con respecto a los tratamientos T2 y T3, en un $8.03 \%$ y $11.50 \%$, respectivamente. Los resultados alcanzados son similares a lo presentado por Izarra (2014), en su estudio "Evaluación de tres sistemas de producción de semilla prebásica en cuatro variedades de papa. Huancavelica, Perú". Pero son inferiores a lo registrado por Quispe (2014), quien realizó un trabajo en la "Evaluación del rendimiento de 10 variedades de papas nativas en la producción de minitubérculos bajo el sistema aeropónico. Andahuaylas, Perú", del cual se puede decir que, el primer trabajo de investigación, está ubicado en un 
escenario con condiciones climáticas muy parecidas al nuestro, obteniendo resultados similares y diferenciándose del siguiente trabajo de investigación, que puede asociarse a aspectos como manejo de la fertilización, composición química del agua y momentos de riego.

De igual manera, en la variable peso del tubérculo por planta, no se encontró diferencia significativa entre los promedios de los tratamientos. Sin embargo, el Tratamiento T2, fue superior numéricamente con respecto a los tratamientos $\mathrm{T} 1$ y T3, con un $3.58 \%$ y un $0.65 \%$ de diferencia, respectivamente. Los resultados alcanzados son similares a lo presentado por Macizo (2013), en su investigación "Comportamiento de dos variedades de papa (Solanum tuberosum L.) para la producción de semilla prebásica según las alternativas en los sistemas clásicos, hidroponía, aeroponía e hidroaeroponía". Sin embargo, los resultados obtenidos son superiores a los registrados por Quispe (2014), en un trabajo de investigación similar. La similitud de los resultados alcanzados con el primer trabajo de investigación, podría deberse a que la altura y temperatura media de ambos escenarios son similares, y difiere del otro ya que las condiciones climáticas de nuestro escenario de investigación permiten obtener valores superiores a lo reportado.

\section{CONCLUSIONES}

Según el análisis del estudio se concluye que la variedad Yungay desarrolla una mayor altura de planta respecto a la variedad Huayro Amazonense, y principalmente, a la variedad Canchán, en la cual se alcanzó la menor altura promedio. Sin embargo, en el número de tubérculos y peso de tubérculos alcanzado en cada variedad, expresaron resultados similares.

Las tres variedades de papa, expresan aptitud para ser empleadas como fuentes de propagación de semillas prebásicas, ya que no existió diferencia significativa entre tratamientos que influenciara para determinar la variedad con mayor y menor rendimiento.

\section{REFERENCIAS BIBLIOGRÁFICAS}

Ahloowalia, B. S. 1999. "Production of Min-Seed Tubers Using a Modular System of Plant Micropropagation." Potato Research 42 (34): 569-575.

Chang, D. C., I. C. Cho, J. T. Suh, S. J. Kim, y Y. B. Lee. 2011. "Growth and Yield Response of Three Aeroponically Grown Potato Cultivars (Solanum tuberosum L.) to Different Electrical Conductivities of Nutrient Solution." American Journal of Potato Research 88 (6): 450-458.

Chang, D. C., C. S. Park, S. Y. Kim, y Y. B. Lee. 2012. "Growth and Tuberization of Hydroponically Grown Potatoes." Potato Research 55 (1): 6981.

Chango, S. J. S. 2012. Producción de Tubérculo Semilla de Papa (Solanum tuberosum), Categoría Prebásica Utilizando Biol en un Sistema Aeropónico en el Cantón Mejía, Provincia de Pichincha. Tesis de Grado. Universidad Ténica de Ambato. Ambato (Ecuador).

Gullino, M .L., A. Camponogara, G. Gasparrini, V. Rizzo, C. Clini y A. Garibaldi. 2003. "Replacing Methyl Bromide for Soil Disinfestation: The Italian Experience and Implications for Other Countries.” Plant Disease 87 (9): 1012 $-1021$.

Hidalgo, O., K. Manrique, C. Velasco, A. Devaux y J. L. Andrade-Piedra. 2009. "Seed Systems for Native Potatoes in Bolivia, Ecuador and Peru: Results of a Diagnostic Study.” En XV Simposio Trianual de la Sociedad Internacional de Cultivos de Raíces Tropicales. Lima (Perú)

Iparraguirre, L. 2017. “Cultivo de Papa Genera 4\% del PBIAgrícola." El Peruano, 7-8

Izarra, F. N. 2014. Evaluación de Tres Sistemas de Produción de Semilla Prebásica en Cuatro Variedades de Papa (Solanum tuberosum L.) INIA-Huancayo. Tesis de Grado. Universidad de Huancavelica. Huancavelica (Perú).

Macizo, R. 2013. Comportamiento de Dos Variedades 
de Papa (Solanum tuberosum L.) para la Producción de Semilla Pre Básica Según las Alternativas en los Sistemas Clásicos, Hidroponía, Aeroponía e Hidroaeroponía. Tesis de Grado. Universidad de Huancavelica. Huancavelica (Perú).

Quispe, S. M. 2014. "Evaluación del Rendimiento de 10 Variedades de Papas Nativas en la Producción de Minitubérculos Bajo el Sistema Aeropónico." Tesis de Grado. Univerisdad Nacional de San Antonio Abad del Cusco. Andahuaylas (Perú).

Ranalli, P. 1997. "Innovative propagation methods in seed tuber multiplication programmes." Potato Research 40 (4): 439-453.

Ritter y Relloso. 2000. "Sistema aeroponico en la producción de patata de siembra de categoría pre básica.” En Congreso Iberoamericano de Investigación y Desarrollo en Patata. VitoriaGasteiz(España).

Velásquez, J. 2006. "Producción de TubérculoSemilla de Papa en la Estación Experimental Santa Catalina del INIAP y su Relación con el Sector Semillero Nacional.” En II Congreso Ecuatoriano de la Papa. Ambato (Ecuador). 